\title{
Mitochondrial function in skeletal muscle of patients with protracted critical illness and ICU-acquired weakness
}

\author{
Kateřina Jiroutková ${ }^{*}$, Adéla Krajčová ${ }^{1,2}$, Jakub Ziak', Michal Fric ${ }^{4}$, Petr Waldauff, Valér Džupa ${ }^{3}$, Jan Gojda ${ }^{2}$,
} Vlasta Němcova-Fürstová, Jan Kováŕr ${ }^{5}$, Moustafa Elkalaf', Jan Trnka ${ }^{1}$ and František Duška ${ }^{1,6}$

\begin{abstract}
Background: Mitochondrial damage occurs in the acute phase of critical illness, followed by activation of mitochondrial biogenesis in survivors. It has been hypothesized that bioenergetics failure of skeletal muscle may contribute to the development of ICU-acquired weakness. The aim of the present study was to determine whether mitochondrial dysfunction persists until protracted phase of critical illness.

Methods: In this single-centre controlled-cohort ex vivo proof-of-concept pilot study, we obtained vastus lateralis biopsies from ventilated patients with ICU-acquired weakness $(n=8)$ and from age and sex-matched metabolically healthy controls $(n=8)$. Mitochondrial functional indices were measured in cytosolic context by high-resolution respirometry in tissue homogenates, activities of respiratory complexes by spectrophotometry and individual functional capacities were correlated with concentrations of electron transport chain key subunits from respiratory complexes II, III, IV and V measured by western blot.

Results: The ability of aerobic ATP synthesis (OXPHOS) was reduced to $\sim 54 \%$ in ICU patients ( $p<0.01$ ), in correlation with the depletion of complexes III ( $\sim 38 \%$ of control, $p=0.02)$ and IV ( $26 \%$ of controls, $p<0.01)$ and without signs of mitochondrial uncoupling. When mitochondrial functional indices were adjusted to citrate synthase activity, OXPHOS and the activity of complexes I and IV were not different, whilst the activities of complexes II and III were increased in ICU patients 3 -fold $(p<0.01)$ respectively 2 -fold $(p<0.01)$.

Conclusions: Compared to healthy controls, in ICU patients we have demonstrated a $\sim 50 \%$ reduction of the ability of skeletal muscle to synthetize ATP in mitochondria. We found a depletion of complex III and IV concentrations and relative increases in functional capacities of complex II and glycerol-3-phosphate dehydrogenase/complex III.
\end{abstract}

\section{Background}

Generalized inflammation and multi-organ failure in the acute phase of critical illness are accompanied by impairment of mitochondrial morphology [1] and function of skeletal muscle [2-5] and other organs [6, 7]. The extent of mitochondrial functional impairment correlates with disease severity, intracellular ATP depletion and outcomes [2]. It appears that the inability to meet cellular ATP demand is caused by a global depletion of functional mitochondria, as the reduction of respiratory complex content [4] or activities [3] is proportional to

\footnotetext{
*Correspondence: katka.nova@post.cz

'Laboratory of Bioenergetics, Third Faculty of Medicine, Charles University in

Prague, Ruská 87, Prague 100 00Prague 10, Czech Republic

Full list of author information is available at the end of the article
}

the reduction of citrate synthase activity (a measure of mitochondrial content) with the exception of septic nonsurvivors, in whom a disproportional reduction of complex I activity has been demonstrated [2]. Moreover, the ability to replenish functional mitochondria is an independent predictor of survival of critical illness [4].

Little is known about mitochondrial function in patients who do survive the acute phase of disease, but fail to wean from mechanical ventilation and enter a protracted phase of critical illness. We hypothesized that bioenergetics failure would be present in the skeletal muscle of patients with weaning failure and ICUacquired weakness as a result of mitochondrial uncoupling and/or depletion. We performed muscle biopsies in such patients, measured concentrations and activities of 
key proteins of the respiratory chain and assessed mitochondrial function in the cytosolic context by highresolution respirometry in fresh skeletal muscle homogenates [8].

\section{Methods}

\section{Overview of study design}

We performed vastus lateralis muscle biopsies in eight patients with protracted critical illness and in eight metabolically healthy control subjects undergoing hip replacement surgery. From the sample (150-200 mg) we prepared a homogenate, which was divided into two parts: the first part was immediately used for respirometry analysis (Protocols 1 and 2), whilst the second part was mixed in 1:1 with a lysis buffer and protease inhibitor, deeply frozen and kept at $-80{ }^{\circ} \mathrm{C}$ for subsequent analysis of respiratory complex individual concentrations (by western blot) and activities (by spectrophotometry).

\section{Study subjects}

Study subjects (age $66.6 \pm 6.6$ years, proportion male/ female $5 / 3$, body mass index (BMI) $27.1 \pm 5.4$ ) were recruited in a general ICU with 22 ventilated beds and a 10-bed medical ICU at Kralovske Vinohrady University Hospital in Prague. Control subjects (age 61.4 \pm 15.8 years, proportion male/female 4/4, BMI $26.6 \pm 3.1$ ) were age-matched metabolically healthy patients undergoing elective hip replacement surgery for degenerative disease, in the Department of Orthopedics of the same hospital. All patients gave prospective informed consent. In patients unable to sign the form due to muscle weakness, the consent procedure was witnessed and assented by the next of kin. The University Hospital Ethical Review Board reviewed both the protocol and the consent form and approved the study. We included patients who had been ventilator-dependent for more than 2 weeks and scored $<48$ points in the Medical Research Council (MRC) score of muscle weakness [9] (scale 0-60 points where 0 means most severe weakness and 60 normal muscle power, an objective measure of muscle weakness). We excluded patients with pre-existing neurological disease, those with severe coagulopathy (platelets $<50 \mathrm{G} / \mathrm{L}$ or international normalized ratio (INR) >1.5) precluding muscle biopsy and patients receiving steroids in higher than substitution doses. Out of 22 eligible ICU patients approached, only 8 consented for muscle biopsy.

Characteristics of study subjects are given in Table 1. Further details about the clinical course of their critical illness preceding the biopsy, including nutrition and glucose control, are in Additional file 1.

\section{Muscle biopsies and sample treatment}

Unless stated otherwise, all chemicals were obtained from Sigma-Aldrich (St Louis, MO). For a full detailed description of the methods, a list of media and buffers and the step-by-step protocol, see Additional file 1.

Muscle biopsies were taken by $5 \mathrm{~mm}$ Bergstrom needle [10] from the vastus lateralis muscle approximately $10 \mathrm{~cm}$ above the knee. In order to minimize patients' discomfort, biopsies from ICU patients were taken under brief general anesthesia, which was required for a routine clinical procedure unrelated to the study (e.g., changing a central line). The sample was collected into $5 \mathrm{~mL}$ of relaxing solution BIOPS containing $10 \mathrm{mM} \mathrm{CaK}_{2}$ EGTA, $7.23 \mathrm{mM} \mathrm{K}$-EGTA, $20 \mathrm{mM}$ imidazole, $20 \mathrm{mM}$ taurine, $50 \mathrm{mM}$ K-MES, $0.5 \mathrm{mM}$ dithiothreitol, $6.56 \mathrm{mM}$ $\mathrm{MgCl}_{2}, 5.77 \mathrm{mM}$ ATP and $15 \mathrm{mM}$ phosphocreatine adjusted to $\mathrm{pH}$ 7.1. The biopsies were kept on ice until further processing.

\section{High-resolution respirometry on skeletal muscle homogenates}

High-resolution respirometry uses polarographic measurement of oxygen consumption by a Clark electrode. This method has been adapted to tissue homogenates

Table 1 Study subject characteristics

\begin{tabular}{|c|c|c|c|c|c|c|c|}
\hline Subject & Diagnosis & Age & APACHE ॥ & Biopsy day & MRC score & LOS-ICU, days & Survived \\
\hline 1 & Septic shock, bronchopneumonia & 70 & 22 & 15 & 20 & 34 & N \\
\hline 2 & Aspiration pneumonia & 80 & 15 & 29 & 23 & 71 & Y \\
\hline 3 & Sepsis & 60 & 31 & 40 & 25 & 92 & N \\
\hline 4 & Cardiogenic shock & 65 & 27 & 41 & 4 & 45 & Y \\
\hline 5 & $\mathrm{CHF}+\mathrm{CAP}$ & 68 & 10 & 27 & 8 & 30 & Y \\
\hline 6 & Chest trauma + HAP & 62 & 14 & 17 & 18 & 48 & Y \\
\hline 7 & CABG, Gl bleed & 68 & 23 & 25 & 16 & 43 & Y \\
\hline 8 & CAP & 60 & 15 & 30 & 23 & 35 & Y \\
\hline Mean \pm SD & & $67 \pm 7$ & $20 \pm 7$ & $28 \pm 9$ & $17 \pm 8$ & $50 \pm 21$ & - \\
\hline
\end{tabular}

Survival means survival to discharge from hospital. APACHE II Acute physiology and chronic health evaluation II score; MRC Medical Research Council score of muscle power, LOS ICU length of stay in intensive care, CHF congestive heart failure, CAP community-acquired pneumonia, CABG coronary artery bypass grafting, $H A P$ hospital-acquired pneumonia, Gl gastrointestinal, $N$ no, $Y$ yes 
[11] including those obtained from human skeletal muscle needle biopsy samples and validated against permeabilized muscle fibers [12] and isolated mitochondria [8]. In brief, connective tissue, fat and blood vessels were gently removed; the skeletal muscle fibers were dried by gauze and weighed on a calibrated scale (= wet weight, Ww). After addition of $\mathrm{K}$ media $(1 \mathrm{~mL} / 100 \mathrm{mg}$ of muscle $\mathrm{Ww}$ ), muscle fibers were homogenized by 4-6 strokes in the Elvhjem-Potter teflon/glass homogeniser. Respirometry was performed at $30{ }^{\circ} \mathrm{C}$ without preoxygenation with $0.2 \mathrm{~mL}$ of $10 \%$ homogenate and $1.9 \mathrm{~mL}$ of $\mathrm{K}$ media in the respirometer Oxygraph $2 \mathrm{~K}$ (Oroboros Instruments, Innsbruck, Austria). K medium contains TrisHCl $(10 \mathrm{mM}), \mathrm{KCl}(80 \mathrm{mM}), \mathrm{MgCl}_{2}(3 \mathrm{mM})$, $\mathrm{KH}_{2} \mathrm{PO}_{4} \quad(5 \mathrm{mM})$, ethylenediaminetetraacetic acid (EDTA) $(1 \mathrm{mM})$, BSA $(0.5 \mathrm{mg} / \mathrm{ml})$ and water at $\mathrm{pH} 7.4$. Oxygen concentrations were kept above a predetermined K90 at all times (See Figure S1 in Additional file 1). Two assays were performed in parallel in two chambers of the respirometer by serial addition of substrates and inhibitors with a Hamilton pipette.

Protocol 1 (see Fig. 1): analysis of global mitochondrial functional indices on homogenates was performed by serial addition of malate $(2.5 \mathrm{mM})+$ glutamate $(15 \mathrm{mM})$, ADP $(1 \mathrm{mM})$, cyt c $(10 \mu \mathrm{M})$, succinate $(10 \mathrm{mM})$, oligomycin $(1 \mu \mathrm{M})$, FCCP $(0.7 \mu \mathrm{M})$, and antimycin $\mathrm{A}(4 \mu \mathrm{M})$. Non-mitochondrial respiration was oxygen consumption measured after addition of antimycin A and subtracted from other values. Capacity of oxidative phosphorylation (OXPHOS, or $3 p$ respiration) was oxygen consumption rate when substrates for both complexes I (malate, glutamate) and II (succinate), abundant ADP and cytochrome c were present. The respiratory chain capacity (state $3 \mathrm{u}$ ) was measured after uncoupling with FCCP. ATP synthesis rate was defined as the decrease in oxygen consumption after addition of oligomycin when substrates for complex I and II were present. The addition of cytochrome $\mathrm{c}$ allows for testing preservation of outer mitochondrial membrane integrity during homogenisation, with values $<20 \%$ considered acceptable [13]. In our subjects the values were $13 \pm 6 \%$ in ICU and $11 \pm 8 \%$ in control patients.

Protocol 2: functional analysis of individual respiratory complexes. We used serial additions (final concentrations in respirometry chamber) of malate $(2.5 \mathrm{mM})$ and glutamate $(15 \mathrm{mM})$; ADP $(1 \mathrm{mM})$; cytochrome c $(10 \mu \mathrm{M})$ rotenone $(3 \mu \mathrm{M})$, succinate $(10 \mathrm{mM})$, malonate (5 mM), glycerol-3-phosphate $(5 \mathrm{mM})$, antimycin A (4 $\mu \mathrm{M})$, ascorbic acid $(10 \mathrm{mM})$ and tetramethyl-pphenylenediamine (TMPD, $200 \mu \mathrm{M})$ and $\mathrm{KCN}(1 \mathrm{mM})$. Complex I activity was calculated as the decrease in oxygen consumption after its inhibitor rotenone, complex II activity as a decrease after addition of malonate. Complex III/glycerol-3-phosphate dehydrogenase (GPDH) activity was determined as an increase of oxygen consumption after addition of glycerol-3-phosphate after both complexes I and II had been inhibited by rotenone and malonate, respectively. Complex IV activity was measured as the increase of oxygen consumption after addition of complex IV substrates ascorbate/TMPD after complex III had been inhibited by antimycin A. See Fig. S2, Additional file 1.

Spectrophotometric analysis of individual activities of respiratory complexes has been described in detail elsewhere [14]. In brief, frozen sample was thawed and homogenized and then exposed to three further cycles of rapid freezing thawing. Complex I assay was performed in an assay mixture composed of $25 \mathrm{mM}$ potassium phosphate, $3.5 \mathrm{~g} / \mathrm{l}$ BSA, $2 \mathrm{mM}$ EDTA, $60 \mu \mathrm{M}$ dichlorophenollindophenol (DCIP), $70 \mu \mathrm{M}$ decylubiquinone, $1 \mu \mathrm{M}$ antimycin $\mathrm{A}$ and $0.2 \mathrm{mM}$ reduced nicotinamide adenine dinucleotide (NADH), $\mathrm{pH}$ 7.8. Changes in absorbance were followed at $600 \mathrm{~nm}$. Rotenone sensitive activity was calculated by subtracting the activity of wells with $10 \mu \mathrm{M}$ rotenone. Complex II activity was measured in an assay mixture containing $80 \mathrm{mM}$ potassium

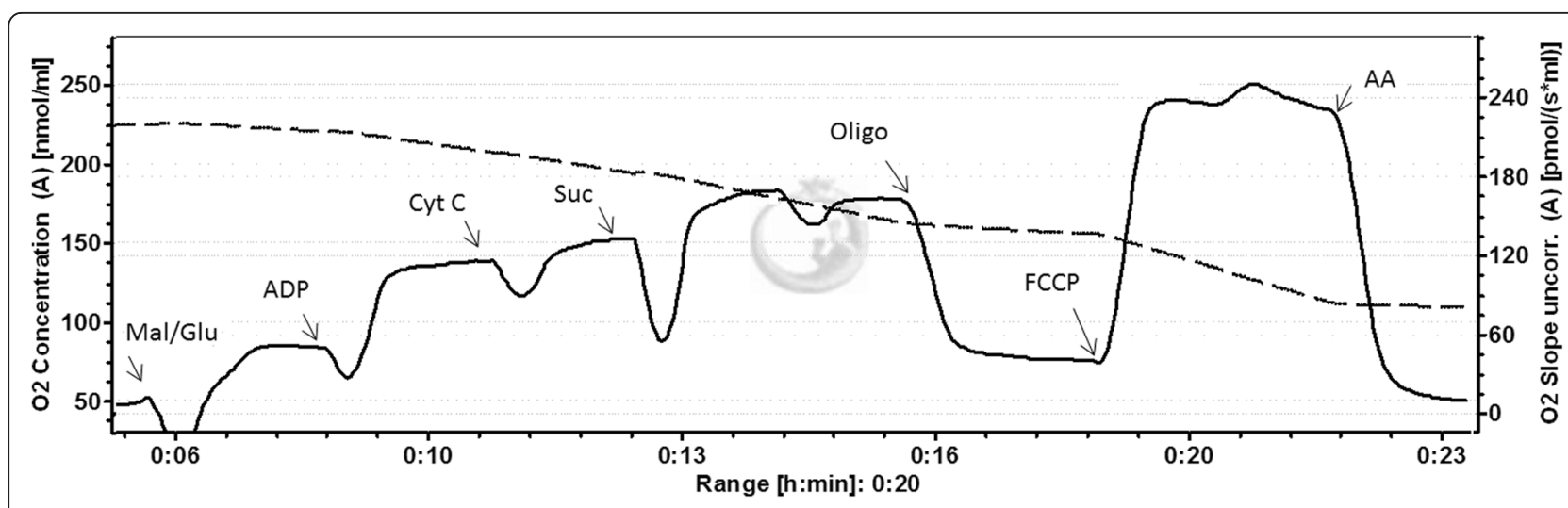

Fig. 1 An example of high-resolution respirometry assay in a homogenate of skeletal muscle, Protocol 1. Solid line represents oxygen consumption rate, dashed line oxygen concentration. Mal/Glu malate/glutamate, suc succinate, oligo oligomycin, FCCP uncoupler, AA antimycin A 
phosphate, $1 \mathrm{~g} / \mathrm{l} \mathrm{BSA}, 2 \mathrm{mM}$ EDTA, $10 \mathrm{mM}$ succinate, $80 \mu \mathrm{M}$ DCIP, $50 \mu \mathrm{M}$ decylubiquinone, $1 \mu \mathrm{M}$ antimycin $\mathrm{A}$ and $3 \mu \mathrm{M}$ rotenone, $\mathrm{pH}$ 7.8. Changes in absorbance were followed at $600 \mathrm{~nm}$. Malonate sensitive activity was calculated by subtracting the activity of wells with $20 \mathrm{mM}$ malonate. Complex III activity was measured in an assay mixture containing $50 \mu \mathrm{M}$ ferricytochrome c, $25 \mathrm{mM}$ potassium phosphate, $4 \mathrm{mM}$ sodium azide, $0.1 \mathrm{mM}$ EDTA, $0.025 \%$ Tween 20 and $50 \mu \mathrm{M}$ decylubiquinol, pH 7.4. Changes in absorbance were followed at $550 \mathrm{~nm}$. Antimycin A sensitive activity was calculated by subtracting the activity of wells with $10 \mu \mathrm{M}$ antimycin A. Complex IV activity was measured in an assay buffer containing $30 \mathrm{mM}$ potassium phosphate and $25 \mu \mathrm{M}$ of freshly prepared ferrocytochrome c, $\mathrm{pH}$ 7.4. Changes in absorbance were followed at $550 \mathrm{~nm}$. The absorbance of samples oxidized with $10 \mu \mathrm{l}$ of $0.5 \mathrm{M}$ potassium hexacyanoferrate (III) was subtracted from all measurements, and then the natural logarithm absorbance was plotted against time and compared to untreated control. Citrate synthase activity was measured using a commercial kit from Sigma, as per manufacturer's instructions [14].

\section{Western blots}

Samples containing $6 \mu \mathrm{g}$ of proteins were mixed with sample buffer and denatured by heating at $45{ }^{\circ} \mathrm{C}$ for 15 minutes. SDS-PAGE and western blotting were performed as described previously [15]. Briefly, proteins were separated on $12 \%$ polyacrylamide gels at $120 \mathrm{~V}$ and then blotted onto a $0.2 \mu \mathrm{m}$ nitrocellulose membrane (Protran BA83, Schleicher-Schuell, Dassel, Germany) for $3 \mathrm{~h}$ at $0.25 \mathrm{~A}$. The membranes were blocked in $5 \%$ weight/volume BSA in Tris-buffered saline for $30 \mathrm{mi}$ nutes at room temperature. The washed membranes were probed with primary antibody cocktail Anti-human Total OxPhos Complex Kit at $4{ }^{\circ} \mathrm{C}$ overnight (dilution 1:175, \# 458199, Life Technologies), containing primary antibodies against complex I (18 kDa), complex II (29 kDa), complex III (core 2; $48 \mathrm{kDa}$ ), complex IV (cytochrome c oxidase (COX) II subunit, $22 \mathrm{kDa}$ ) and $\mathrm{F}_{1} \mathrm{~F}_{\mathrm{O}}$ ATPase $(\mathrm{F} 1 \alpha ; 45 \mathrm{kDa})$ subunits. After washing, the membranes were incubated for $2 \mathrm{~h}$ at room temperature with mouse horseradish peroxidase-conjugated secondary antibody (dilution 1:6600; Santa Cruz Biotechnology, Santa Cruz, CA, USA). Protein bands were visualized with an enhanced chemiluminescence detection system (Thermo Fisher Scientific, Rockford, IL, USA) using Carestream Gel Logic 4000 PRO Imaging System (Carestream Health, New Haven, CT, USA). To demonstrate equal loading, the membrane was stripped and reprobed with anti-GAPDH antibody (dilution 1:1000, \# ab9485, Abcam, Cambridge, UK). Densitometry was performed using the Carestream v5.2 program (Carestream Health). Data were normalized to glyceraldehyde 3- phosphate dehydrogenase (GAPDH) and referenced to an internal standard (a control patient sample was present on every immunoblot).

\section{Statistics}

Data are presented as median (interquartile range). The Man-Whitney $U$ test was used for all comparisons. Statistica 8.0 (StatSoft Inc., USA) was used for all calculations and $\mathrm{p}<0.05$ was considered statistically significant.

\section{Results \\ Relative content of mitochondrial proteins}

In ICU patients compared to controls, there was a significant reduction of core 2 subunit of complex III (median content in ICU patients was approximately $38 \%$ of that in controls, $p=0.02$ ) and COX2 subunit of complex IV (approximately $26 \%, p<0.01$ ). No differences were detected in subunits of $\mathrm{F}_{1} \mathrm{~F}_{\mathrm{O}}$ ATPase (approximately $109 \%, p=0.89$ ) or complex II (approximately $90 \%$, $p=0.99$ ). (see Fig. 2). We were unable to determine the content of subunits of complex I (the signals were bellow detection limits in both ICU and control patients).

\section{Global indices of mitochondrial function in skeletal muscle homogenates (Protocol 1)}

In the skeletal muscle of patients with protracted critical illness (ICU) compared to control subjects (control), there was a reduction in citrate synthase (CS) activity per muscle wet weight (median 0.25 (IQR $0.16-0.28$ ) vs 0.34 (IQR 0.28-0.43) nkat/mg Ww, $p=0.03$ ). In keeping with this, the capacity of OXPHOS and of the respiratory chain were significantly reduced in ICU patients (approximately $54 \%$ and $52 \%$ of that in controls, $p<0.01$ and $p=0.03$ ) when expressed per muscle wet weight. OXPHOS normalized to muscle wet weight was correlated with the activity of CS $\left(r^{2}=0.53, p=0.01\right)$, the content of COX II subunit of respiratory complex IV $\left(r^{2}=0.39, p=0.03\right)$ and there was a trend towards a correlation to core 2 subunit of complex III $\left(r^{2}=0.29\right.$, $p=0.06$ ), but no relations at all were seen to concentrations of complex II $\left(r^{2}=0.04, p=0.50\right)$ or $\mathrm{F}_{1 \alpha}$ subunit of $\mathrm{F}_{1} \mathrm{~F}_{\mathrm{O}}$ ATPase $\left(r^{2}=0.02, p=0.67\right)$.

After adjustment to CS activity, the differences in mitochondrial functional indices between ICU patients and control subjects disappeared (see Table 2). Of note, there was no difference in the degree of uncoupling of inner mitochondrial membrane between ICU patients and controls.

\section{Analysis of function of individual respiratory complexes}

Protocol 2: by using sequential addition of substrates and specific inhibitors of the respiratory chain complexes, we were able to determine maximum electron 

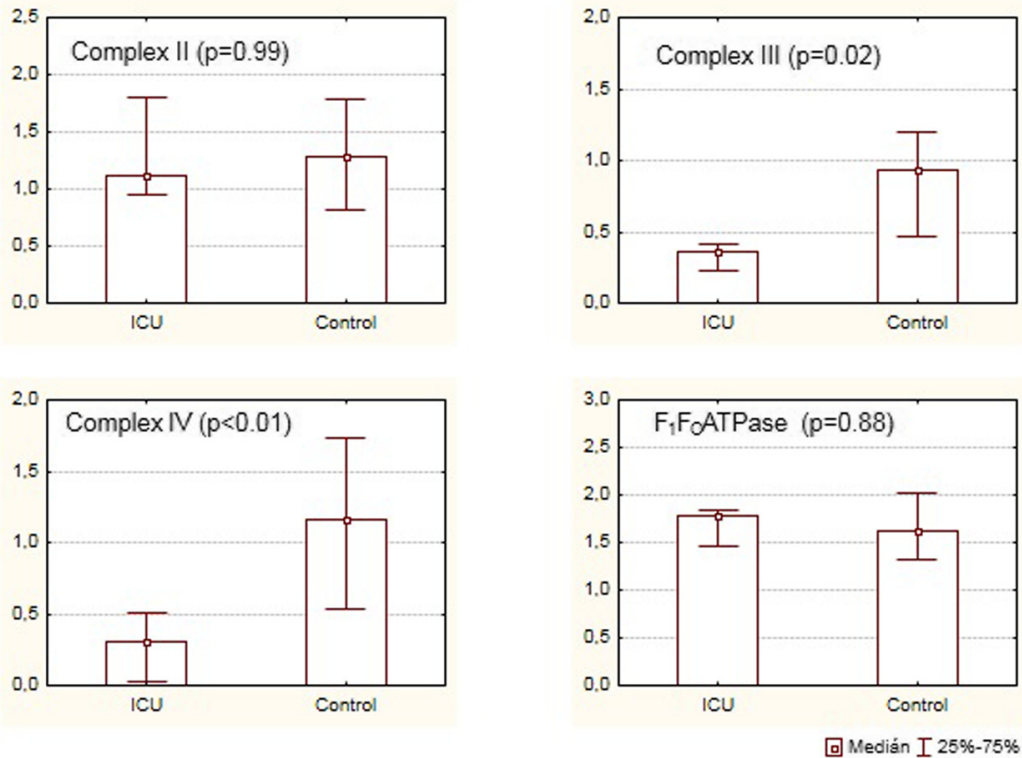

\section{ICU}

Control

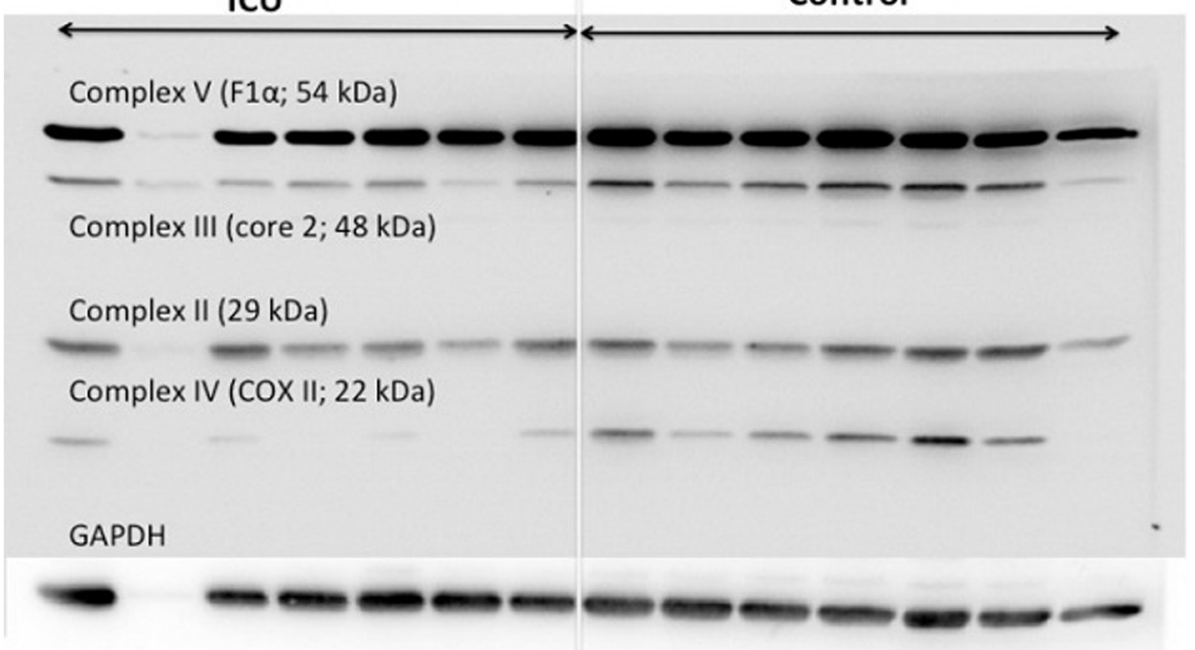

Fig. 2 Concentrations of functional subunits of respiratory complexes in arbitrary units and an example of an immunoblot membrane. Data are presented as medians, vertical bars represent interquartile ranges. GAPDH glyceraldehyde 3-phosphate dehydrogenase, COX cytochrome c oxidase

fluxes through them. Oxygen consumption rates were adjusted to the activity of CS (see Fig. 3, upper row). Functional capacity of complexes I and IV were not different between ICU patients and the control group. Surprisingly, the capacity of complex II was demonstrated in the ICU group to be approximately $300 \%$ of the capacity in controls $(p<0.01)$. The capacity of complex III/ GPDH was also significantly $(p<0.01)$ higher in ICU patients as compared to controls. Very similar results were obtained when the individual activity of respiratory complexes was measured by spectrophotometry (Fig. 3).

Last we asked whether there is a relationship between the capacity of OXPHOS (or state $3 p$ as determined in Protocol 1) and specific functional capacity of individual respiratory complexes (as determined in Protocol 2). Complex I $\left(r^{2}=0.33, p=0.04\right)$, and even more strongly complex IV $\left(r^{2}=0.65, p<0.01\right)$ correlated to OXPHOS, whilst complexes II and III/GPDH did not $\left(r^{2}=0.08\right.$, $p=0.36$ and $r^{2}=0.12, p=0.28$, respectively). See Fig. S3 in the Supplementary material.

\section{Discussion}

This study is the first to demonstrate mitochondrial dysfunction in skeletal muscle of patients with protracted critical illness. In the skeletal muscles of these patients we observed approximately $50 \%$ reduction in the ability to synthetize ATP by aerobic phosphorylation per mg of muscle wet weight $\left(\mathrm{OXPHOS} / \mathrm{W}_{\mathrm{w}}\right)$ which correlated 
Table 2 Mitochondrial functional indices measured by high-resolution respirometry in homogenates

\begin{tabular}{|c|c|c|c|c|c|c|c|}
\hline \multirow{2}{*}{\multicolumn{2}{|c|}{ Parameter }} & \multicolumn{3}{|c|}{ Per muscle wet weight (pmol/s.mg Ww) } & \multicolumn{3}{|c|}{ Per CS activity (pmol.nkat-1.s-1) } \\
\hline & & $\mathrm{ICU}(\mathrm{n}=7)$ & Control $(n=8)$ & P & $\mathrm{ICU}(\mathrm{n}=7)$ & Control $(n=8)$ & P \\
\hline \multicolumn{2}{|c|}{ OXPHOS (3p) } & $7.6(5.0-8.8)^{*}$ & $13.9(11.3-17.9)$ & $<0.01$ & $31(28-36)^{*}$ & $37(32-74)$ & 0.15 \\
\hline \multicolumn{2}{|c|}{ RC capacity $(3 \mathrm{u})$} & $8.6(6.7-10.5)$ & $16.4(13.0-20.6)$ & 0.03 & $41(37-44)$ & $42(37-98)$ & 0.46 \\
\hline \multicolumn{2}{|c|}{ Non-mito OCR } & $0.8(0.6-1.5)$ & $0.8(0.6-1.3)$ & 0.91 & $4(3-5)$ & $2(1-4)$ & 0.16 \\
\hline \multirow[t]{2}{*}{$\mathrm{F}_{1} \mathrm{~F}_{\mathrm{o}}$ ATPase } & Absolute & $6.1(4.8-7.6)^{*}$ & $12.6(9.2-13.0)$ & $<0.01$ & $26(26-30)^{*}$ & $33(29-49)$ & 0.46 \\
\hline & $\%$ OXPHOS & $81(77-83)^{*}$ & $84(80-89)$ & 0.36 & $81(77-83)^{*}$ & $84(80-89)$ & 0.36 \\
\hline \multirow[t]{2}{*}{ Proton leak } & Absolute & $1.3(1.0-1.4)^{*}$ & $2.2(1.3-3.6)$ & 0.10 & $8(5-9)^{*}$ & $7(4-11)$ & 0.95 \\
\hline & $\%$ OXPHOS & $19(17-23)^{*}$ & $16(11-20)$ & 0.36 & $19(17-23)^{*}$ & $16(11-20)$ & 0.36 \\
\hline \multicolumn{2}{|l|}{ Complex I } & $4.8(4.0-6.1)$ & $6.7(5.5-8.6)$ & 0.19 & $23(22-35)$ & $23(18-26)$ & 0.35 \\
\hline \multicolumn{2}{|l|}{ Complex II } & $4.6(2.9-6,5)$ & $1.5(0.8-3.8)$ & 0.06 & $23(20-28)$ & $8(3-14)$ & $<0.01$ \\
\hline \multicolumn{2}{|c|}{ Complex III/ GPDH } & $1.5(1.1-1.9)$ & $0.8(0.4-1.3)$ & 0.12 & $7.4(6.0-9.3)$ & $1.8(1.2-3.9)$ & $<0.01$ \\
\hline \multicolumn{2}{|l|}{ Complex IV } & $15.5(13.0-19.5)$ & $19.7(15.3-27.5)$ & 0.30 & 88 (69-99) & $49(40-113)$ & 0.12 \\
\hline
\end{tabular}

Data presented as median (interquartile range), $p$ value as per Mann-Whitney $U$ test. ${ }^{*} \mathrm{~N}=5$ for ICU patients. GPDH glycerol-3-phosphate dehydrogenase, Non-mito $O C R$ non-mitochondrial oxygen consumption rate, OXPHOS oxidative phosphorylation, $R C$ respiratory chain

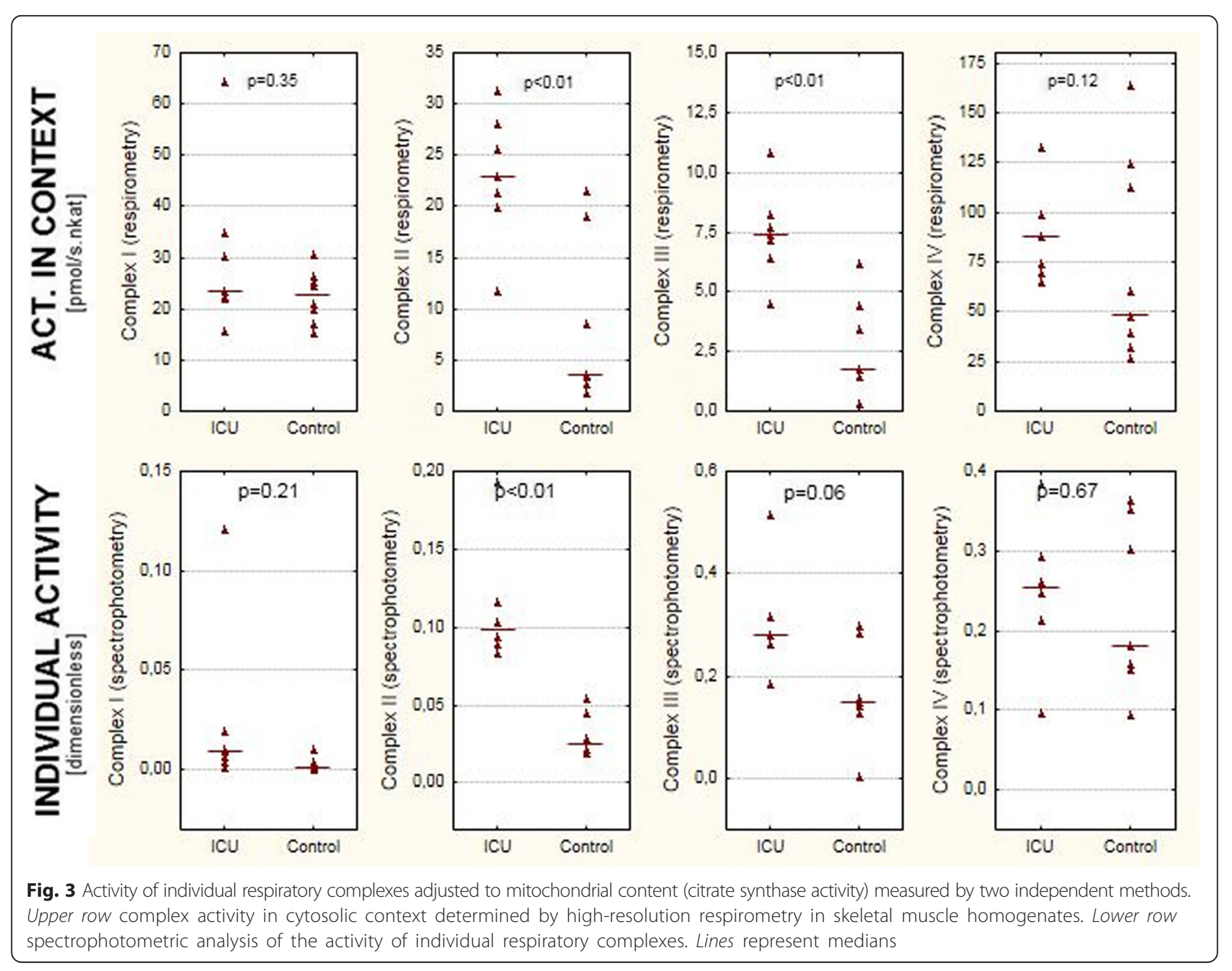


with the concentration of depleted complex IV. Complex III was also depleted, unlike complexes II and V. When OXPHOS was adjusted to citrate synthase activity (OXPHOS/CS), the differences between ICU patients and control subjects disappeared and OXPHOS/ $/ \mathrm{W}_{\mathrm{w}}$ strongly correlated with citrate synthase activity. The obvious interpretation of these results is that mitochondria are depleted in ICU patients, whilst complexes II and V are relatively abundant in remaining functional mitochondria. A similar disproportionality of the concentrations of respiratory complexes has been described in skeletal muscle during aging [16] and oxidative stress [17]. Even though citrate synthase activity is widely used as a marker of mitochondrial content $[2,18-20]$, it may become a subject of oxidative damage [21] and therefore it may not reliably reflect the mitochondrial density. Because we have not used an alternative method of measuring mitochondrial content (e.g., electron microscopy), we cannot say whether the depletion of complexes III and IV occurred in isolation or as part of mitochondrial depletion. It is the concentration of the depleted complex IV (and possibly complex I) that was limiting for the mitochondrial function, in keeping with data of Levy [22], who demonstrated the relation of complex IV dysfunction to bioenergetics failure in acute sepsis. Contrary to our hypothesis, there was no sign of increased mitochondrial uncoupling in ICU patients.

In order to explore the functional capacity of individual complexes, we performed a respirometry protocol in which we used specific substrates and inhibitors of individual complexes. If expressed per muscle wet weight (Table 2), we saw a trend towards increase in functional capacity of respiratory complexes II and III, whilst that of complexes I and IV tended to be non-significantly reduced to approximately $70 \%$ of values seen in control subjects, and correlated with OXPHOS. After adjustment for citrate synthase activity, complexes II and III were increased significantly (threefold and twofold respectively, $p<0.01$ ) and complexes I and IV were not different (Fig. 3). High-resolution respirometry measures the changes in oxygen consumption in fresh intact tissue homogenates after addition of respiratory substrates and inhibitors [11]. The sample contains intact mitochondria in a cytosolic context and it is believed that this approach better reflects physiological alterations occurring in vivo [23]. The technique has been calibrated against permeabilized muscle fibers [12] and isolated mitochondria [8]. When using this method for measuring the functional capacity of individual complexes one must bear in mind that the rate-limiting step can in theory appear downstream of the complex that is being analyzed. Complexes III and IV are under physiological conditions able to accommodate the flux of electrons from both complexes I and II and it is therefore unlikely that they become rate-limiting when fed by electrons from either complex I or II in isolation. For testing complex III we used glycerol-3-phosphate as a substrate whilst complexes I and II had been blocked. By doing so we avoided the risk of downstream limitation (i.e., at complex IV), but on the other hand, the rate-limiting step may be at the level of GPDH, which is functionally a part of the glycerol phosphate shuttle rather than the respiratory chain.

With these limitations of respirometry in mind, we repeated the measurements of individual complex activities by a different technique. Classical spectrophotometry is a well-established method $[2,3,18]$, which assesses the activities of respiratory complexes by using artificial complex-specific substrates after the organelle structure has been destroyed by repeated freezing and thawing. This means that the measured activity of each complex is independent of the functionality of other complexes. As demonstrated in Fig. 3, both methods gave very similar results and confirmed the increased functional capacity of complexes II and III/GPDH in the critically ill as compared to control subjects.

Complex II (succinate dehydrogenase) normally drives electrons from succinate oxidation to fumarate in the citric acid cycle (CAC) via flavin adenine dinucleotide (FAD) to the respiratory chain. CAC itself is heavily dependent on reoxidation of NADH by complex I as it produces three molecules of NADH per one molecule of $\mathrm{FADH}_{2}$. Eventual increase in NADH/NAD+ ratio inhibits CAC. Similarly, aerobic glycolysis produces $2 \mathrm{NADH} / \mathrm{mol}$ ecule of glucose during the conversion to pyruvate and a further 2NADH by converting pyruvate to acetyl-CoA, which is oxidised in CAC. However, during oxidation of fatty acid and carbon skeletons of branched chain amino acids, reduced coenzymes $\mathrm{FADH}_{2}$ and NADH are produced in a 1:1 ratio. Of all catabolic pathways, fatty acid oxidation is thus least dependent on the functionality of complex I. In the acute phase of critical illness complex I seems to be predominantly impaired [2] and upregulation of complex II at a later stage can be a compensatory response or an attempt to bypass dysfunctional complex I. Insulin resistance is a well-known feature of critical illness $[24,25]$ and it has been shown that GLUT-4 dependent transport is dysfunctional in patients with ICUAW (weakness developing in a critically ill patient without an identifiable cause other than nonspecific inflammation) [26] and pyruvate dehydrogenase is inhibited [27]. Skeletal muscle in protracted critical illness thus may suffer from starvation of carbohydrate-derived substrate for CAC. On the contrary, free fatty acids are elevated in the critically ill $[24,28]$ and intracellular lipid droplets accumulate early in diaphragmatic and biceps muscle in brain-dead donors [18]. Branched-chain amino acids (BCAA) derived from muscle protein degradation are deaminated in skeletal 
muscle and their carbons are oxidized in a similar way to fatty acid oxidation. Relative upregulation of complex II in the context of mitochondrial dysfunction may thus represent an adaptive response to insulin resistance [29] and preferential oxidation of lipids and BCAA over carbohydrates. Glycerol-3-phosphate can be formed from glycerol derived from lipolysis [30], and it requires respiratory complexes distal to complex I to be converted to glyceraldehyde-3-phosphate [31], a glycolytic intermediate. Upregulation of complex III/GPDH seen in our ICU patients may reflect the increase in intracellular lipid turnover in the skeletal muscle of these patients.

However, the lack of correlation between OXPHOS and both functional capacities and relative abundance of complexes II and III/GPDH suggests that they may play other functions, which are not directly related to aerobic ATP production. It has been recently shown that cells accumulate succinate during hypoxia [32-34] or inflammation [35]. When oxygenation is restored, rapid reoxidation of succinate produces electron flux, which downstream complexes are unable to absorb, and which is redirected backwards to complex I, generating excessive amounts of reactive oxygen species [36, 37]. Relative redundancy of the activity of complexes II and III over complex I observed by us in protracted illness could be an adaptation against cell damage when intracellular succinate levels are fluctuating.

Indeed our study has many limitations. First, our data are derived from a small group of highly selected subjects. We found it very difficult to consent patients for the biopsy in this non-therapeutic study. With such a small number of subjects there is always a risk of type II error, i.e., that we were unable to detect changes that were present. High inter-individual variability in the concentration and functionality of respiratory complexes (see Fig. 3) is well-known [2, 22], and further complicates the interpretation of data. Biopsies were performed in ICU patients who had been ventilatordependent for more than 2 weeks (mean 28 days) and suffered from muscle weakness. We have selected this cohort of patients with muscle dysfunction in order to maximize the chances of observing any alteration of bioenergetics in a non-respiratory muscle, which seems to be less affected, even in the acute phase of critical illness, when compared to the diaphragm $[18,38]$ or intercostal muscles [3]. As a result, it remains unclear whether the changes in mitochondrial metabolism described above are consequences of prolonged immobility [39-41], the critical illness, or whether they occur only in patients who are weak. Of note, our control subjects were ambulatory elective hip surgery patients and it is unknown whether their potentially reduced mobility affected the mitochondrial function of skeletal muscle. In light of this, our pilot study should be treated as a proof-of-concept study and the results interpreted with caution.

\section{Conclusions}

In conclusion, we have demonstrated mitochondrial dysfunction in the quadriceps muscle of patients with protracted critical illness compared to metabolically healthy age-matched control patients undergoing hip replacement surgery. There was approximately $50 \%$ reduction in the capacity for aerobic ATP synthesis per $\mathrm{mg}$ of muscle wet weight, in correlation with significant reductions in functional subunits of complexes III and IV. When accounting for the activity of citrate synthase, which we used as a marker of mitochondrial content, there was no difference in global mitochondrial functional indices. We have shown a significant increase in the functional capacity of complexes II and III/GPDH. This can be possibly explained by metabolic adaptation to insulin resistance or succinate fluctuation, but exploring these hypotheses warrants further studies.

\section{Additional file}

Additional file 1: Supplementary material. (DOCX 115 kb)

\section{Abbreviations}

ATP: adenosine triphosphate; BCAA: branched-chain amino acids; BMI: body mass index; BSA: bovine serum albumin; CABG: coronary artery bypass grafting; CAC: citric acid cycle; CAP: community-acquired pneumonia; CHF: congestive heart failure; COX: cytochrome coxidase; CS: citrate synthase; Cyt c: cytochrome c; DCIP: dichlorophenollindophenol; EDTA: ethylenediaminetetraacetic acid; FAD: flavin adenine dinucleotide; FCCP: carbonyl cyanide-4-(trifluoromethoxy)phenylhydrazone;

GAPDH: glyceraldehyde 3-phosphate dehydrogenase; GPDH: glycerol-3phosphate dehydrogenase; HAP: hospital-acquired pneumonia;

ICUAW: Intensive Care Unit - acquired weakness; KDa: kiloDalton; LOS ICU: length of stay in intensive care; MRC: Medical Research Council score of muscle power, $\mathrm{NAD}^{+}$, nicotinamide adenine dinucleotide; OXPHOS: oxidative phosphorylation; TMPD: N,N,N',N'-tetramethyl-p-phenylenediamine; Ww: wet weight.

\section{Competing interests}

The authors declare no competing interests.

\section{Authors' contributions}

$\mathrm{KJ}$ processed muscle samples, participated in the respirometry analysis and contributed to the study design. AK and JZ helped to design the study, processed muscle samples, performed (together with MK) the respirometry analyses. ME and JT (together with AK) performed spectrophotometric analysis of respiratory complexes. VD, MF and JG obtained informed consents and performed the biopsies, whilst VFN and JK carried out the western blots. FD conceived of the study, participated in its coordination and performed the statistical analysis. All authors wrote their parts of the manuscript, revised the first draft and then read and approved the final version of the manuscript.

\section{Acknowledgements}

The study was supported from grants IGA NT 12319 and PRVOUK P31 (coordinator: Prof. Michal Andel). We thank all volunteers who participated in the study and Dr Paul James for proofreading. 


\section{Author details}

${ }^{1}$ Laboratory of Bioenergetics, Third Faculty of Medicine, Charles University in Prague, Ruská 87, Prague 100 00Prague 10, Czech Republic. ${ }^{2}$ Department of Internal Medicine II, Kralovske Vinohrady University Hospital, Praque, Czech Republic. ${ }^{3}$ Department of Orthopaedic Surgery, Kralovske Vinohrady University Hospital, Prague, Czech Republic. ${ }^{4}$ Department of Anaesthesia and Intensive Care, Kralovske Vinohrady University Hospital, Prague, Czech Republic. ${ }^{5}$ Department of Cell and Molecular Biology \& Center for Research of Diabetes, Metabolism and Nutrition, Third Faculty of Medicine, Charles University in Prague, Prague, Czech Republic. ${ }^{6}$ Adult Intensive Care Unit, Queen's Medical Centre, Nottingham University Hospital NHS Trust, Nottingham, UK

\section{Received: 8 September 2015 Accepted: 6 December 2015} Published online: 24 December 2015

\section{References}

1. Takasu O, Gaut JP, Watanabe E, To K, Fagley RE, Sato B, et al. Mechanisms of cardiac and renal dysfunction in patients dying of sepsis. Am J Respir Crit Care Med. 2013;187:509-17.

2. Brealey D, Brand M, Hargreaves I, Heales S, Land J, Smolenski R, et al. Association between mitochondrial dysfunction and severity and outcome of septic shock. Lancet. 2002;360:219-23.

3. Fredriksson $\mathrm{K}$, Hammarqvist $\mathrm{F}$, Strigård $\mathrm{K}$, Hultenby $\mathrm{K}$, Ljungqvist $\mathrm{O}$, Wernerman J, Rooyackers $\mathrm{O}$. Derangements in mitochondrial metabolism in intercostal and leg muscle of critically ill patients with sepsis-induced multiple organ failure. Am J Physiol Endocrinol Metab. 2006;291:E1044-50.

4. Carré JE, Orban J-C, Re L, Felsmann K, Iffert W, Bauer M, et al. Survival in critical illness is associated with early activation of mitochondrial biogenesis. Am J Respir Crit Care Med. 2010;182:745-51.

5. Garrabou G, Morén C, López S, Tobías E, Cardellach F, Miró O, Casademont J. The effects of sepsis on mitochondria. J Infect Dis. 2012;205:392-400

6. Vanhorebeek I, De Vos R, Mesotten D, Wouters PJ, De Wolf-Peeters C, Van den Berghe G. Protection of hepatocyte mitochondrial ultrastructure and function by strict blood glucose control with insulin in critically ill patients. Lancet. 2005;365:53-9.

7. Japiassú AM, Santiago AP, d'Avila JC, Garcia-Souza LF, Galina A, Castro FariaNeto $\mathrm{HC}$, et al. Bioenergetic failure of human peripheral blood monocytes in patients with septic shock is mediated by reduced F1Fo adenosine-5'triphosphate synthase activity. Crit Care Med. 2011;39:1056-63.

8. Ziak J, Krajcova A, Jiroutkova K, Nemcova V, Dzupa V, Duska F. et al. Assessing the function of mitochondria in cytosolic context in human skeletal muscle: Adopting high-resolution respirometry to homogenate of needle biopsy tissue samples. Mitochondrion. 2015;21:106-12.

9. Edwards RHT. Muscle fatigue. Postgrad Med J. 1975;51:137-143. http://pmj bmj.com/content/51/593/137.

10. Hayot M, Michaud A, Koechlin C, Caron MA, Leblanc P, Préfaut C, Maltais F. Skeletal muscle microbiopsy: a validation study of a minimally invasive technique. Eur Respir J. 2005;25:431-40.

11. Pecinová A, Drahota Z, Nůsková $H$, Pecina $P$, Houštěk J. Evaluation of basic mitochondrial functions using rat tissue homogenates. Mitochondrion. 2011;11:722-8

12. Larsen S, Kraunsøe R, Gram M, Gnaiger E, Helge JW, Dela F. The best approach: Homogenization or manual permeabilization of human skeletal muscle fibers for respirometry? Anal Biochem. 2014;446:64-8.

13. Schuh RA, Jackson KC, Khairallah RJ, Ward CW, Spangenburg EE. Measuring mitochondrial respiration in intact single muscle fibers. AJP. 2012;302:R7129.

14. Trnka J, Elkalaf M, Andel M. Lipophilic triphenylphosphonium cations inhibit mitochondrial electron transport chain and induce mitochondrial proton leak. PLoS ONE. 2015;10:e0121837-14.

15. Němcová-Fürstová $V$, James RF, Kovář J. Inhibitory effect of unsaturated fatty acids on saturated fatty acid-induced apoptosis in human pancreatic cells: activation of caspases and ER stress induction. Cell Physiol Biochem. 2011; 27(5):525-38.

16. Hepple RT, Baker DJ, Kaczor JJ, Krause DJ. Long-term caloric restriction abrogates the age-related decline in skeletal muscle aerobic function. FASEB J. 2005:19(10):1320-2.

17. Crane JD, Abadi A, Hettinga BP, Ogborn DI, MacNeil LG, Steinberg GR, et al. Elevated mitochondrial oxidative stress impairs metabolic adaptations to exercise in skeletal muscle. PLoS One. 2013;8(12):e81879.
18. M, Jung B, Liang F, Azuelos I, Hussain S, Goldberg P, et al. Mitochondrial dysfunction and lipid accumulation in the human diaphragm during mechanical ventilation. Am J Respir Crit Care Med. 2012;186:1140-9.

19. Larsen S, Nielsen J, Hansen CN, Nielsen LB, Wibrand F, Stride N, et al. Biomarkers of mitochondrial content in skeletal muscle of healthy young human subjects. J Physiol. 2012;590(Pt 14):3349-60.

20. Wibom R, Hultman E, Johansson M, Matherei K, Constantin-Teodosiu D, Schantz PG. Adaptation of mitochondrial ATP production in human skeletal muscle to endurance training and detraining. J Appl Physiol. 1992;73:2004-10.

21. Chepelev NL, Bennitz JD, Wright JS, Smith JC, Willmore WG. Oxidative modification of citrate synthase by peroxyl radicals and protection with novel antioxidants. J Enzyme Inhib Med Chem. 2009;24(6):1319-31.

22. Levy RJ, Deutschman CS. Cytochrome c oxidase dysfunction in sepsis. Crit Care Med. 2007;35:S468-75.

23. Barrientos A, Fontanesi F, Diiaz F. Evaluation of the Mitochondrial Respiratory Chain and Oxidative Phosphorylation System Using Polarography and Spectrophotometric Enzyme Assays. Hoboken, NJ, USA: John Wiley \& Sons, Inc; 2001

24. Cree MG, Wolfe RR. Postburn trauma insulin resistance and fat metabolism. Am J Physiol Endocrinol Metab. 2008;294:E1-9.

25. Bakalar B, Duska F, Pachl J, Fric M, Otahal M, Pazout J, Andel M. Parenterally administered dipeptide alanyl-glutamine prevents worsening of insulin sensitivity in multiple-trauma patients. Crit Care Med. 2006;34(2):381-7.

26. Weber-Carstens S, Schneider J, Wollersheim T, Assmann A, Bierbrauer J, Marg A, et al. Critical illness myopathy and GLUT4. Am J Respir Crit Care Med. 2013;187:387-96.

27. Vary TC. Sepsis-induced alterations in pyruvate dehydrogenase complex activity in rat skeletal muscle: effects on plasma lactate. Shock. 1996;6:89-94.

28. Duska F, Fric M, Waldauf P, Pazout J, Andel M, Mokrejs P, Tůma P, Pachl J. Frequent intravenous pulses of growth hormone together with glutamine supplementation in prolonged critical illness after multiple trauma: Effects on nitrogen balance, insulin resistance, and substrate oxidation. Crit Care Med. 2008:36(6):1707-13.

29. Lee JS. Saturated, but not n-6 polyunsaturated, fatty acids induce insulin resistance: role of intramuscular accumulation of lipid metabolites. J Appl Physiol. 2006;100:1467-74.

30. Watford M. Functional glycerol kinase activity and the possibility of a major role for glyceroneogenesis in mammalian skeletal muscle. Nutr Rev. 2000;58:145-8.

31. Mráček T, Drahota Z, Houštěk J. The function and the role of the mitochondrial glycerol-3-phosphate dehydrogenase in mammalian tissues. BBA - Bioenergetics. 2013;1827:401-10.

32. Lukyanova LD. Mitochondrial Signaling in Hypoxia. OJEMD. 2013;03:20-32.

33. Chouchani ET, Pell VR, Gaude E, Aksentijević D, Sundier SY, Robb EL, et al. Ischaemic accumulation of succinate controls reperfusion injury through mitochondrial ROS. Nature. 2014;515:431-5.

34. O'Neill L. Succinate strikes. Nature. 2014:515:1-2.

35. Tannahill GM, Curtis AM, Adamik J, Palsson-McDermott EM, McGettrick AF, Goel $G$, et al. Succinate is an inflammatory signal that induces IL-1 $\beta$ through HIF-1a. Nature. 2014;496:238-42.

36. Quinlan CL, Perevoshchikova IV, Hey-Mogensen M, Orr AL, Brand MD. Sites of reactive oxygen species generation by mitochondria oxidizing different substrates. Redox Biol. 2013;1:304-12.

37. Chouchani ET, Methner C, Nadtochiy SM, Logan A, Pell VR, Ding S, et al. Cardioprotection by S-nitrosation of a cysteine switch on mitochondrial complex I. Nat Med. 2013:19:753-9.

38. Peruchi BB, Petronilho F, Rojas HA, Constantino L, Mina F, Vuolo F, et al. Skeletal muscle electron transport chain dysfunction after sepsis in rats. J Surg Res. 2011;167:e333-8

39. Holloszy JO, Coyle EF. Adaptations of skeletal muscle to endurance exercise and their metabolic consequences. J Appl Physiol Respir Environ Exerc Physiol. 1984;56(4):831-8.

40. Berg HE, Dudley GA, Hather B, Tesch PA. Work capacity and metabolic and morphologic characteristics of the human quadriceps muscle in response to unloading. Clin Physiol. 1993;13(4):337-47.

41. Hikida RS, Gollnick PD, Dudley GA, Convertino VA, Buchanan P. Structural and metabolic characteristics of human skeletal muscle following 30 days of simulated microgravity. Aviat Space Environ Med. 1989;60(7):664-70. 